\title{
Macrohongos presentes en un ÁREA de MANEJo RENEgenerativo de bosque de Mata Atlántica (Misiones, Argentina)
}

\author{
EMANUEL M. GRASSI ${ }^{1}$, GONZALO M. ROMANO ${ }^{2}$ y NAHUEL F. SCHENONE 3
}

\begin{abstract}
Summary: Macrofungi present in a recovered zone of Atlantic Forest (Misiones, Argentina). Atlantic Forest is an ecosystem historically managed without restrictions. Over the years, this situation led to a fragmentation of the forest environment with the consequent loss of habitats for all kinds of organisms in the region. The present work is a checklist of macrofungi found in a native forest in Oberá (Misiones, Argentina), where conservation and regeneration practices have been held since 2009 to recover its natural state. Sampling took place in different seasons for 3 years. 136 species have been identified, distributed in 13 orders, 32 families and 86 genera. The objective of the present work was to set a precedent of macrofungi diversity in the area. This will allow studying macrofungi ecological succession and their potential as bio-indicators of forest recovery.
\end{abstract}

Key words: Biodiversity, macrofungi, Paranaense Forest.

Resumen: El Bosque de Mata Atlántica es un ecosistema que histórica y continuamente se ve sometido a manejo forestal desmedido. Esto ha generado una fragmentación del ambiente boscoso con su consecuente pérdida de hábitat para todo tipo de organismos de la región. El objetivo del presente trabajo fue generar un listado de especies de macrohongos presentes en un área de manejo de bosque regenerativo en la zona de Oberá (Misiones, Argentina), donde desde 2009 se llevan a cabo tareas de recuperación y regeneración de biodiversidad del Bosque de Mata Atlántica. Se realizaron muestreos a campo en diferentes estaciones durante 3 años. Como resultado se presenta un catálogo de especies de macrohongos presentes en la zona que consta de 136 especies distribuidas en 13 órdenes, 32 familias y 86 géneros. Este conocimiento permitirá profundizar estudios en distintos grupos de macrohongos y analizar su capacidad como bioindicadores del estado de conservación de la zona de Bosque Atlántico en proceso de recuperación.

Palabras clave: Diversidad, Selva Paranaense, macrohongos.

\section{INTRODUCCIÓN}

La vegetación predominante en la provincia de Bosque Paranaense, subregión del Chaco (Morrone, 2014) es la del bosque subtropical semideciduo, las variaciones en el ambiente local y el tipo de suelo

\footnotetext{
1 DBBE, Facultad de Ciencias Exactas y Naturales, Universidad de Buenos Aires, INMIBO (UBA-CONICET). Intendente Güiraldes 2160, Ciudad Autónoma de Buenos Aires, Argentina. emagrassi@bg.fcen.uba.ar

${ }^{2}$ Departamento de Biología, Facultad de Ciencias Naturales, Universidad Nacional de la Patagonia San Juan Bosco, CONICET. Ruta 259 Km 16, Esquel, Chubut, Argentina.

${ }^{3}$ Centro de Investigaciones Antonia Ramos, Ruta $103 \mathrm{Km}$ 70, Oberá, Misiones, Argentina.
}

permiten la existencia de diferentes comunidades vegetales. La ecorregión posee un clima subtropical. La temperatura media anual es de $19-24^{\circ} \mathrm{C}$, con una variación anual relativamente alta. En la porción sur de la ecorregión, las heladas son comunes en los meses de invierno (junio-agosto), especialmente en las zonas altas. Las precipitaciones en la región varían entre los 1000 y los $2200 \mathrm{~mm}$ anuales; habitualmente son menores en la parte norte de la ecorregión que en la parte sur y no están distribuidas uniformemente a lo largo del año.

Muchos son los estudios de biodiversidad en la región, sin embargo la mayoría se focaliza en las áreas protegidas del Parque Nacional do Iguaçú (Brasil) y el Parque Nacional Iguazú (Argentina), por ejemplo Ryvarden \& de Meijer, (2002) y Lechner et al. (2006), respectivamente. 
En estas áreas protegidas (AP) se han registrado 470 especies de aves (Saibene et al. 1996) y más de 250 especies de árboles. Se han registrado entre 53 y 73 especies arbóreas por hectárea en parcelas de estudio en el Parque Nacional Iguazú (Placci \& Giorgis, 1993). Sólo allí también se han registrado 85 especies de orquídeas, lo que representa un tercio de las especies conocidas para toda la Argentina (Johnson, 2001).

De los trabajos de diversidad de hongos, el más significativo y abarcativo es Wright \& Wright (2005), que mostró una micobiota representada por 46 especies del filo Ascomycota y 374 especies pertenecientes al filo Basidiomycota. Siguiendo la clasificación de Hibbett et al. (2007), del primer filo 5 especies son del orden Pezizales, 10 del orden Hypocreales, 19 del orden Xylariales y 12 de otros Ascomycota. El filo Basidiomycota fue estudiado en mayor detalle e incluye 6 especies del orden Auriculariales, 1 de Dacrymicetales, 4 de Geastrales, 4 de Gomphales, 1 de Phallales, 26 del orden Cantharellales, 61 de Hymenochaetales, 101 de Agaricales, 132 del orden Polyporales y las restantes 38 de otros órdenes.

Los hongos juegan un rol importante en los ecosistemas, centrado en el reciclado del carbono y otros elementos esenciales para otros organismos. Debido a su nutrición heterotrófica, la fuente de energía es captada de la materia que conforman otros organismos, tanto vivos como muertos. Por lo tanto tienen una gran capacidad degradadora que le permite descomponer esa materia orgánica, de origen vegetal, animal o incluso fúngico, para transformarla en compuestos más simples que es devuelta al ecosistema. Entre estos hongos, denominados saprofíticos, se destacan aquellos degradadores de madera (Dix \& Webster, 1995). Los hongos degradadores juegan un rol importante en la diversidad de organismos asociados a la madera, como son los insectos, aves y pequeños mamíferos que utilizan la madera muerta para nidificar y/o obtener alimento (Blackwell \& Jones, 1997; Cockle et al., 2012).

Otro grupo importante de hongos es el de los micorrícicos, que han co-evolucionado junto con las plantas generando asociaciones simbióticas de diferentes tipos (Kiers \& Van der Heijden, 2006). Como producto de dicha simbiosis los hongos micorrícicos reciben directamente de las plantas los azúcares que precisan para desarrollarse. A cambio captan del suelo y ceden a sus hospedantes vegetales los nutrientes minerales y el agua que éstos necesitan para crecer (Smith \& Read, 2008; van der Heijden et al. 2015). Dado que la gran mayoría de las plantas en el mundo forman al menos un tipo de asociación micorrícica, y la cantidad de recursos que son movilizados entre hongos y plantas a través de estas asociaciones, los hongos micorrícicos tienen un rol significativo en el ciclo de nutrientes global (van der Heijden et al. 2015).

La extracción de madera debido al desarrollo de la industria forestal genera una alteración de la biota asociada a ella, empezando por los hongos. Esta alteración de la micobiota asociada a los bosques es un indicador del estado de degradación en que se encuentra el bosque (Nordén et al. 2004). El manejo de bosques y plantaciones forestales suele conllevar un aumento en el número y riqueza de especies de hongos que crecen sobre la madera (Romano et al. 2014).

Por este motivo es de particular interés estudiar zonas en donde se realizan tareas de conservación y regeneración de biodiversidad que sirvan de base para futuros estudios. La zona en la que se han realizado los muestreos cuenta con una superficie de aproximadamente 700 hectáreas donde se desarrollan diversas actividades de conservación y regeneración de Bosque de Mata Atlántica, principalmente de reforestación con árboles nativos como Handroanthu simpetiginosus (Mart. ex DC.) Mattos (lapacho rosado), Handroanthus albus (Cham.) Mattos (lapacho amarillo), Albizzia hassleri (Chodat) Burkart (anchico blanco), Cedrela fissilis Vell. (cedro misionero), Apuleia leiocarpa (Vogel) J.F.Macbr. (grapia), Campomanesia pubescens (DC.) O. Berg (guabiroba), Enterolobium contortisiliquum (Vell.) Morong (timbó), Bauhinia ungulata L. (escalera de mono), entre otras. También se está trabajando con la reinserción de mamíferos como Leopardus pardalis (ocelote), Dasypus novemcinctus (peludo), Euphractus sexcinctus (Mulita) y Leopardus tigrinus (gato tirica), entre otros.

El presente trabajo refleja el relevamiento estacional de la micobiota hallada en la zona de manejo regenerativo; se remarca la necesidad de ampliar los estudios en zonas que no estén protegidas legalmente, pero que puedan poseer una riqueza de especies que no debe ser desestimada. 


\section{Material y Método}

El área de estudio está localizada en el Municipio de Campo Ramón, $27^{\circ} 26^{\prime}$ S y $54^{\circ} 55^{\prime}$ O, departamento de Oberá, provincia de Misiones (Argentina) (Fig. 1). Se encuentra a una altitud de entre 300-500 m.s.n.m entre las cuencas de los arroyos Acaraguá y Arroyo del Medio, rodeado de selvas subtropicales de estacionalidad térmica periódica, con una variación hidrológica no tan predecible como la temperatura. Se realizaron 6 campañas durante 3 años en distintas estaciones (12/2011; 06/2012; 01/2013; 08/2013; 03/2014; 07/2014).

Las clasificaciones taxonómicas se basan en el trabajo de Hibbett et. al. (2007). Para las determinaciones taxonómicas se trabajó con bibliografía específica para cada grupo: Agaricales (Singer \& Digilio 1951; Singer 1986; Niveiro et al. 2010; Niveiro et al. 2014), Polyporales (Cunningham, 1965; Eriksson \& Ryvarden, 1973; Ryvarden \& Johansen 1980; Corner 1983, 1984, 1987, 1989; Gilbertson \& Ryvarden 1986; Gilbertson \& Ryvarden 1987; Ryarden 1991, 2004; Ryvarden \& Gilbertson 1994; Robledo \& Rajchenberg, 2007), Fomes (Lowe, 1957), Corticiaceae (Bernicchia \& Gorjon, 2010), Phallales (Calonge, 1998), Hymenochaetaceae (Larsen \& Cobb-Poulle, 1990) y Xylariales (Hladki \& Romero, 2005; Hladki et al. 2009; Hladki \& Romero, 2010).

Todos los esporomas maduros de especies de los distintos grupos de macrohongos fueron recolectados, secados y analizados como se detalla en Mata Hidalgo et al. (2009).

\section{Resultados}

El presente listado (Tabla 1) está conformado por 136 especies, de las cuales la mayoría pertenecen a la familia Polyporaceae (37), seguida por la familia Xylariaceae (18) e Hymenochaetaceae (8). Tres órdenes se destacan en el estudio por ser los de mayor número de especies registradas (Tabla 2). En el filo Ascomycota se destaca el orden Xylariales representado por la familia Xylariaceae aportando un $13.23 \%$ de especies al estudio (Tabla 2).

Dentro del filo Basidiomycota se destacan el $55^{\circ} 38^{\prime} \mathrm{O}$ $55^{\circ} 50^{\prime} \mathrm{O}$

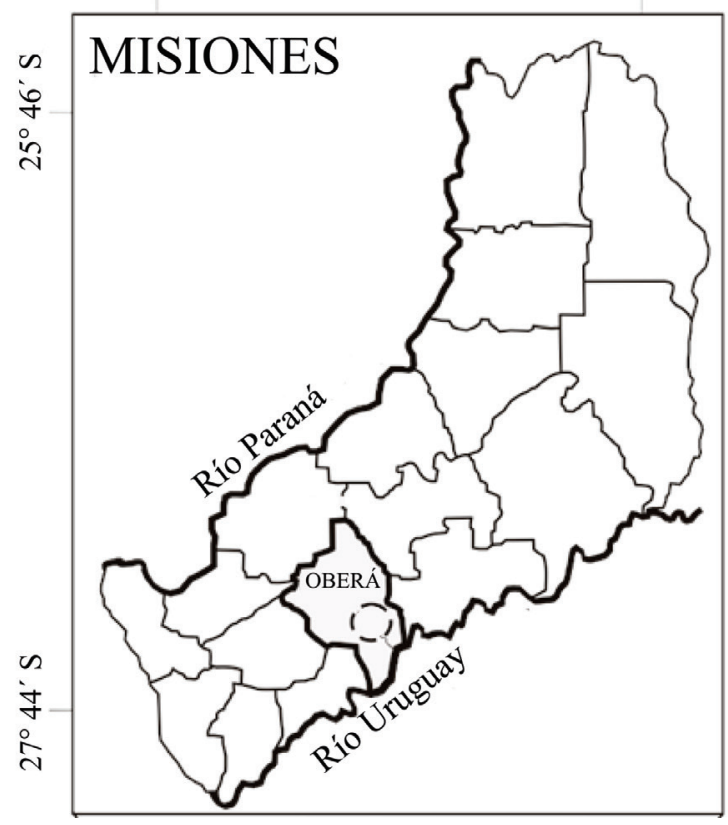

Fig. 1. Mapa de la provincia de Misiones, el círculo punteado indica el área de muestreo relevada (modificado de Avigliano \& Schenone 2015).

orden Polyporales representado por 5 familias y el orden Agaricales representado por 13 familias. A nivel de especie, el orden Polyporales posee la mayor riqueza (54) distribuida en 33 géneros y 5 familias. El orden Agaricales ha sido el de mayor cantidad de familias encontradas (13), dentro de las cuales se reparten 25 géneros y 32 especies (Tabla 2).

Los géneros con mayor número de especies son Polyporus (9), Xylaria (6), Marasmius (5), Geastrum (5) y Ganoderma (5), los cuales poseen distintos hábitos degradadores: de madera (Polyporus, Xylaria y Ganoderma), de hojarasca (Marasmius) y humícolas (Geastrum).

\section{Discusión}

En Misiones, la mayoría de estudios de biodiversidad fúngica se han centrado en el Parque Nacional Iguazú (Wright \& Wright, 2005) y en la Reserva de Biosfera Yabotí (Niveiro et al. 2010), 
Bol. Soc. Argent. Bot. 51 (2) 2016

Tabla 1. Listado de especies identificadas en la zona de Oberá (Misiones, Argentina)

\begin{tabular}{|c|c|c|c|}
\hline Filo & Orden & Familia & Nombre científico \\
\hline \multirow{29}{*}{ Ascomycota } & Helotiales & Helotiaceae & Bisporella citrina (Batsch) Korf \& S.E. Carp. 1974 \\
\hline & \multirow{5}{*}{ Hypocreales } & Cordycipitaceae & Cordyceps militaris (L.) Fr. 1818 \\
\hline & & \multirow{3}{*}{ Ophiocordycipitaceae } & Ophiocordyceps australis (Speg.) G.H. Sung et. al. 2007 \\
\hline & & & $\begin{array}{l}\text { Ophiocordyceps curculionum (Tul. \& C. Tul.) G.H. Sung et. } \\
\text { al. } 2007\end{array}$ \\
\hline & & & Ophiocordyceps unilateralis (Tul. \& C. Tul.) Petch 1931 \\
\hline & & Nectriaceae & Nectria cinnabarina (Tode) Fr. 1849 \\
\hline & \multirow{5}{*}{ Pezizales } & Pyronemataceae & Scutellinia scutellata (L.) Lambotte (1887) \\
\hline & & \multirow{4}{*}{ Sarcoscyphaceae } & Cookeina colensoi (Berk.) Seaver 1913 \\
\hline & & & Cookeina tricholoma (Mont.) Kuntze 1891 \\
\hline & & & Phillipsia domingensis Berk. 1881 \\
\hline & & & Rickiella edulis (Speg.) Pfister 1987 \\
\hline & \multirow{18}{*}{ Xylariales } & \multirow{18}{*}{ Xylariaceae } & $\begin{array}{l}\text { Annulohypoxylon stygium (Lév.) Y.M. Ju, J.D. Rogers \& H.M. } \\
\text { Hsieh } 2005\end{array}$ \\
\hline & & & $\begin{array}{l}\text { Annulohypoxylon nitens (Ces.) Y.M. Ju, J.D. Rogers \& H.M. } \\
\text { Hsieh } 2005\end{array}$ \\
\hline & & & $\begin{array}{l}\text { Camillea fossulata (Mont.) Læssøe, J.D. Rogers \& Whalley } \\
1989\end{array}$ \\
\hline & & & Entonaema liquescens Möller 1901 \\
\hline & & & Hypoxylon julianii L.E. Petrini 1986 \\
\hline & & & Hypoxylon lenormandii Berk. \& M.A. Curtis 1868 \\
\hline & & & Hypoxylon monticulosum Mont. 1856 \\
\hline & & & Hypoxylon rubiginosum (Pers.) Fr. 1849 \\
\hline & & & $\begin{array}{l}\text { Phylacia turbinata (Berk.) Speg. } 1889 \\
\text { Obs.: Cita nueva para la Argentina }\end{array}$ \\
\hline & & & Poronia oedipus (Mont.) Mont 1856 \\
\hline & & & Rosellinia necatrix Berl. ex Prill. 1904 \\
\hline & & & Rosellinia subiculata (Schwein.) Sacc. 1882 \\
\hline & & & Xylaria arbuscula Sacc. 1878 \\
\hline & & & Xylaria cubensis (Mont.) Fr. 1851 \\
\hline & & & Xylaria enteroleuca (Speg.) P.M.D. Martin 1970 \\
\hline & & & Xylaria hypoxylon (L.) Grev. 1824 \\
\hline & & & Xylaria grammica (Mont.) Mont. 1851 \\
\hline & & & Xylaria polymorpha (Pers.) Grev. 1824 \\
\hline
\end{tabular}


E. M. Grassi et al. - Macrohongos de la zona centro de Misiones

\begin{tabular}{|c|c|c|c|}
\hline Filo & Orden & Familia & Nombre científico \\
\hline \multirow{35}{*}{ Basidiomycota } & \multirow{32}{*}{ Agaricales } & \multirow{4}{*}{ Agaricaceae } & Cyathus stercoreus (Schwein.) De Toni 1888 \\
\hline & & & Cyathus striatus (Huds.) Willd. 1787 \\
\hline & & & Leucocoprinus tenellus (Boud.) Locq. 1943 \\
\hline & & & Lycoperdon sp. \\
\hline & & Bolbitiaceae & Pholiota filaris (Fr.) Singer 1936 \\
\hline & & \multirow{2}{*}{ Entolomataceae } & Entoloma howellii (Peck) Dennis 1953 \\
\hline & & & Entoloma permutatum E. Horak 1978 \\
\hline & & \multirow{6}{*}{ Marasmiaceae } & Marasmius androsaceus var. androsaceus (L.) Fr. 1838 \\
\hline & & & Marasmius cladophyllus Berk. 1856 \\
\hline & & & Marasmius crinis-equi F. Muell. ex Kalchbr. 1880 \\
\hline & & & Marasmius haematocephalus (Mont.) Fr. 1838 \\
\hline & & & Marasmius eucladopus Singer 1965 \\
\hline & & & Tetrapyrgos subdendrophora (Redhead) E. Horak 1987 \\
\hline & & \multirow{3}{*}{ Mycenaceae } & Mycena spinosissima (Singer) Desjardin 1995 \\
\hline & & & Mycena alphitophora (Berk.) Sacc. 1887 \\
\hline & & & Xeromphalina tenuipes (Schwein.) A.H. Sm. 1953 \\
\hline & & Omphalotaceae & Marasmiellus coilobasis (Berk.) Singer 1973 \\
\hline & & Physalacriaceae & Oudemansiella platensis (Speg.) Speg. 1881 \\
\hline & & Pleurotaceae & Pleurotus albidus (Berk.) Pegler 1983 \\
\hline & & Pluteaceae & Pluteus xylophilus (Speg.) Singer 1949 \\
\hline & & \multirow{2}{*}{ Psathyrellaceae } & Coprinellus sp. \\
\hline & & & Parasola sp. \\
\hline & & Schizophyllaceae & Schizophyllum commune Fr. 1815 \\
\hline & & \multirow{5}{*}{ Strophariaceae } & Agrocybe neocoprophila Singer 1953 \\
\hline & & & Ciclocybe cylindracea (DC.) Vizzini \& Angelini 2014 \\
\hline & & & Gymnopilus lepidotus Hesler 1969 \\
\hline & & & Psilocybe cubensis (Earle) Singer 1948 \\
\hline & & & Stropharia coronilla (Bull.) Quél. 1872 \\
\hline & & \multirow{4}{*}{ Tricholomataceae } & Amparoina spinosissima (Singer) Singer 1958 \\
\hline & & & Arrhenia epichysium (Pers.) Redhead et al. 2002 \\
\hline & & & Clytocybe sp. \\
\hline & & & Leucopaxillus gracillimus Singer \& A.H. Sm. 1943 \\
\hline & \multirow{3}{*}{ Auriculariales } & \multirow{3}{*}{ Auriculariaceae } & Auricularia mesenterica (Dicks.) Pers. 1822 \\
\hline & & & Auricularia fuscosuccinea (Mont.) Henn. 1893 \\
\hline & & & Auricularia nigricans (Fr.) Birkebak et al. 2013 \\
\hline
\end{tabular}


Bol. Soc. Argent. Bot. 51 (2) 2016

\begin{tabular}{|c|c|c|c|}
\hline Filo & Orden & Familia & Nombre científico \\
\hline \multirow{35}{*}{ Basidiomycota } & Dacrymicetales & Dacrymycetaceae & Dacryopinax spathularia (Schwein.) G.W. Martin 1948 \\
\hline & Cantharellales & Botryobasidiaceae & $\begin{array}{l}\text { Botryobasidium vagum (Berk. \& M.A. Curtis) D.P. } \\
\text { Rogers } 1935\end{array}$ \\
\hline & \multirow{6}{*}{ Geastrales } & \multirow{6}{*}{ Geastraceae } & Geastrum lageniforme Vittad. 1842 \\
\hline & & & Geastrum javanicum Lév. 1846 \\
\hline & & & Geastrum saccatum Fries, 1829. \\
\hline & & & Geastrum triplex Jungh. 1840 \\
\hline & & & Geastrum violaceus Rick 1906 \\
\hline & & & Sphaerobolus sp. \\
\hline & Gomphales & Gomphaceae & Ramaria sp. \\
\hline & \multirow{8}{*}{ Hymenochaetales } & \multirow{8}{*}{ Hymenochaetaceae } & Fomitiporia neotropica Campos-Santana et al. 2014 \\
\hline & & & Hymenochaete curtisii (Berk.) Morgan 1888 \\
\hline & & & Hymenochaete damicornis (Link) Léveillé 1846 \\
\hline & & & Phellinus fastuosus (Lév.) S. Ahmad 1972 \\
\hline & & & Phellinus gilvus (Schwein.) Pat. 1900 \\
\hline & & & Phellinus merrillii (Murrill) Ryvarden 1972 \\
\hline & & & Phylloporia sp. \\
\hline & & & $\begin{array}{l}\text { Tropicoporus linteus (Berk. \& M.A. Curtis) L.W. Zhou \& } \\
\text { Y.C. Dai } 2015\end{array}$ \\
\hline & Phallales & Phallaceae & Phallus indusiatus Vent. 1798 \\
\hline & \multirow{17}{*}{ Polyporales } & \multirow[b]{2}{*}{ Fomitopsidaceae } & Auriporia brasilica G. Coelho 2005 \\
\hline & & & $\begin{array}{l}\text { Fomitopsis palustris (Berk. \& M.A. Curtis) Gilb. \& } \\
\text { Ryvarden } 1985\end{array}$ \\
\hline & & \multirow{6}{*}{ Ganodermataceae } & Amauroderma boleticeum (Pat. \& Gaillard) Torrend 1920 \\
\hline & & & Ganoderma australe (Fr.) Pat. 1889 \\
\hline & & & Ganoderma applanatum (Persoon) Patouillard 1887 \\
\hline & & & Ganoderma lipsiense (Batsch) G.F. Atkinson \\
\hline & & & Ganoderma lucidum (Curtis) P. Karst. 1881 \\
\hline & & & Ganoderma stipitatum (Murrill) Murrill 1908 \\
\hline & & \multirow{3}{*}{ Meripilaceae } & Hydnopolyporus fimbriatus (Cooke) D.A. Reid 1962 \\
\hline & & & Rigidoporus ulmarius (Sowerby) Imazeki 1952 \\
\hline & & & Rigidoporus vinctus (Berk.) Ryvarden 1972 \\
\hline & & \multirow{6}{*}{ Meruliaceae } & $\begin{array}{l}\text { Cymatoderma caperatum (Berk. \& Mont.) D.A. Reid } \\
(1956)\end{array}$ \\
\hline & & & Junghuhnia polycystidifera (Rick) Rajchenb. 1987 \\
\hline & & & Mycoacia uda (Fr.) Donk 1931 \\
\hline & & & Phlebia tremelloidea (Bres.) Parmasto 1967 \\
\hline & & & Phlebia subulata J. Erikss. \& Hjortstam 1981 \\
\hline & & & $\begin{array}{l}\text { Steccherinum reniforme (Berk. \& M.A. Curtis) Banker } \\
1906\end{array}$ \\
\hline
\end{tabular}


E. M. Grassi et al. - Macrohongos de la zona centro de Misiones

\begin{tabular}{|c|c|c|c|}
\hline Filo & Orden & Familia & Nombre científico \\
\hline \multirow{36}{*}{ Basidiomycota } & \multirow{36}{*}{ Polyporales } & \multirow{36}{*}{ Polyporaceae } & Coriolopsis floccosa (Jungh.) Ryvarden 1972 \\
\hline & & & Datronia mollis (Sommerf.) Donk 1966 \\
\hline & & & Datronia scutellata (Schwein.) Gilb. \& Ryvarden 1985 \\
\hline & & & Favolus tenuiculus P. Beauv. 1806 \\
\hline & & & Fomes fasciatus (Sw.) Cooke 1885 \\
\hline & & & Funalia caperata (Berk.) Zmitr. \& V. Malysheva 2013 \\
\hline & & & Fuscocerrena portoricensis (Fr.) Ryvarden 1982 \\
\hline & & & Grammothele subargentea (Speg.) Rajchenb. 1983 \\
\hline & & & Grammothele fuligo (Berk. \& Broome) Ryvarden 1979 \\
\hline & & & Hexagonia hydnoides (Sw.) M. Fidalgo 1968 \\
\hline & & & Lentinus berteroi (Fr.) Fr. 1825 \\
\hline & & & Lentinus velutinus Fr. 1830 \\
\hline & & & Lentinus swartzii Berk. 1843 \\
\hline & & & Lenzites elegans (Spreng.) Pat. 1900 \\
\hline & & & Megasporoporia setulosa (Henn.) Rajchenb. 1982 \\
\hline & & & Mycobonia flava (Sw.) Pat. 1894 \\
\hline & & & Nigroporus vinosus (Berk.) Murrill 1905 \\
\hline & & & Pachykytospora alabamae (Berk. \& Cooke) Ryvarden 1972 \\
\hline & & & Panus similis (Berk. \& Broome) T.W. May \& A.E. Wood 1995 \\
\hline & & & Perenniporia contraria (Berk. \& M.A. Curtis) Ryvarden 1972 \\
\hline & & & Perenniporiella neofulva (Lloyd) Decock \& Ryvarden 2003 \\
\hline & & & Polyporus arcularioides A. David \& Rajchenb. 1985 \\
\hline & & & Polyporus arcularius (Batsch) Fr. 1821 \\
\hline & & & Polyporus ciliatus Fr. 1815 \\
\hline & & & Polyporus dictyopus Mont. 1835 \\
\hline & & & Polyporus guianensis Mont. 1840 \\
\hline & & & Polyporus leprieurii Mont. 1840 \\
\hline & & & Polyporus tricholoma Mont. 1837 \\
\hline & & & Polyporus brumalis (Pers.) Fr. 1818 \\
\hline & & & Polyporus varius (Pers.) Fr. 1821 \\
\hline & & & Pseudofavolus orinocensis (Pat. \& Gaillard) Ryvarden 1972 \\
\hline & & & Pycnoporus sanguineus (L.) Murrill 1904 \\
\hline & & & Tinctoporellus epimiltinus (Berk. \& Broome) Ryvarden 1979 \\
\hline & & & Trametes pavonia (Berk.) Fr. 1851 \\
\hline & & & Trametes versicolor (L.) Lloyd 1921 \\
\hline & & & Trametes villosa (Sw.) Kreisel 1971 \\
\hline
\end{tabular}


Bol. Soc. Argent. Bot. 51 (2) 2016

Tabla 2. Número de géneros y especies de macrohongos detectados y agrupados por orden en un área de manejo regenerativo de bosque Atlántico de la provincia de Misiones.

\begin{tabular}{|c|c|c|c|c|c|}
\hline Filo & Orden & Familia (\# especies) & $\begin{array}{l}\text { Géneros } \\
\text { por orden }\end{array}$ & $\begin{array}{l}\text { Especies } \\
\text { por orden }\end{array}$ & $\begin{array}{c}\% \text { de especies } \\
\text { aportado }\end{array}$ \\
\hline \multirow{7}{*}{ Ascomycota } & Helotiales & Helotiaceae (1) & 1 & 1 & 0,74 \\
\hline & \multirow{3}{*}{ Hypocreales } & Cordycipitaceae (1) & \multirow{3}{*}{3} & \multirow{3}{*}{5} & \multirow{3}{*}{3,67} \\
\hline & & Ophiocordycipitaceae (3) & & & \\
\hline & & Nectriaceae (1) & & & \\
\hline & \multirow{2}{*}{ Pezizales } & Pyronemataceae (1) & \multirow{2}{*}{4} & \multirow{2}{*}{5} & \multirow{2}{*}{3,67} \\
\hline & & Sarcoscyphaceae (4) & & & \\
\hline & Xylariales & Xylariaceae (18) & 8 & 18 & 13,23 \\
\hline \multirow{25}{*}{ Basidiomycota } & \multirow{13}{*}{ Agaricales } & Agaricaceae (4) & \multirow{13}{*}{25} & \multirow{13}{*}{32} & \multirow{13}{*}{23,53} \\
\hline & & Bolbitiaceae (1) & & & \\
\hline & & Entolomataceae (2) & & & \\
\hline & & Marasmiaceae (6) & & & \\
\hline & & Mycenaceae (3) & & & \\
\hline & & Omphalotaceae (1) & & & \\
\hline & & Physalacriaceae (1) & & & \\
\hline & & Pleurotaceae (1) & & & \\
\hline & & Pluteaceae (1) & & & \\
\hline & & Psathyrellaceae (2) & & & \\
\hline & & Schizophyllaceae (1) & & & \\
\hline & & Strophariaceae (5) & & & \\
\hline & & Tricholomataceae (4) & & & \\
\hline & Auriculariales & Auriculariaceae (3) & 1 & 3 & 2,21 \\
\hline & Dacrymycetales & Dacrymycetaceae (1) & 1 & 1 & 0,74 \\
\hline & Cantharellales & Botryobasidiaceae (1) & 1 & 1 & 0,74 \\
\hline & Geastrales & Geastraceae (6) & 2 & 6 & 4,41 \\
\hline & Gomphales & Gomphaceae (1) & 1 & 1 & 0,74 \\
\hline & Hymenochaetales & Hymenochaetaceae (8) & 5 & 8 & 5,87 \\
\hline & Phallales & Phallaceae (1) & 1 & 1 & 0,74 \\
\hline & \multirow{5}{*}{ Polyporales } & Fomitopsidaceae (2) & \multirow{5}{*}{33} & \multirow{5}{*}{54} & \multirow{5}{*}{39,71} \\
\hline & & Ganodermataceae (6) & & & \\
\hline & & Meripilaceae (3) & & & \\
\hline & & Meruliaceae (6) & & & \\
\hline & & Polyporaceae (37) & & & \\
\hline Total & 13 & 32 & 86 & 136 & 100 \\
\hline
\end{tabular}


tratando este último solo las especies de Agaricales sensu Singer (1986). La inclusión de nuevos sitios de estudio permite tener un conocimiento cada vez más abarcativo de la micobiota presente en ecosistemas nativos. Este es el primer estudio sobre diversidad de hongos en la zona centro de la provincia de Misiones. Si bien el número total de especies es menor respecto del trabajo de Wright \& Wright (2005), los autores realizaron en este trabajo un checklist sobre material de herbarios.

Entre las especies encontradas se destacan Phylacia turbinata, nueva cita para la Argentina (Silva et al. 2015) y Rickiella edulis, que posee un ascoma efímero que solo fue registrado en la Argentina dos veces, siendo la tercera vez que se lo encuentra en el país (Vignale et al. 2015).

La presencia de material ligninolítico en el suelo del bosque es un factor importante para asegurar un flujo de nutrientes desde los árboles hacia los microorganismos que componen el ecosistema, posibilitando la regeneración de la biodiversidad perdida en sitios afectados por prácticas de extracción maderera (Fridman \& Walheim, 2000). El material ligninolítico (ramas, troncos, hojas) cumplen el rol de mantener en su estructura componentes como carbono y nitrógeno que durante la degradación son liberados haciéndolos nuevamente biodisponibles para la flora, fauna y demás organismos del ambiente (Nordén et al. 2004). Esta degradación es llevada a cabo por hongos como los macrohongos.

Los procesos extractivos de madera dan lugar a una disminución de la diversidad de hongos, especialmente de los Ascomycota y degradadores de madera, grupos que en el pasado se han descuidado en los trabajos de conservación (Nordén et al. 2004). Por consiguiente, la diversidad fúngica es un parámetro que debe ser considerado en zonas en proceso de regeneración, dado que la riqueza específica es un indicador vital del estado del bosque.

En la zona donde se desarrolló el trabajo, se realizan tareas de regeneración desde 2009, llegando actualmente a 100.000 árboles de diferentes especies nativas en 700 hectáreas. Se destaca que el $40 \%$ de las especies registradas corresponden al orden Polyporales (Tabla 2), todas degradadoras de la madera. Es necesario aumentar los esfuerzos de muestreo en zonas en procesos de regeneración, y generar líneas de base de hongos.
El estudio de la biodiversidad y ecología de hongos de una zona necesariamente comienza por el relevamiento de las especies presentes en el lugar. Esto permite conocer la micobiota con la que se cuenta como línea de base para realizar distintos estudios ecológicos y poder comparar con otros sitios que hayan sido previamente caracterizados.

\section{Agradecimientos}

Los autores desean agradecer a la Fundación Bosques Nativos Argentinos para la Biodiversidad (FBNA) y el Centro de Investigaciones Antonia Ramos (CIAR) por permitirnos realizar las campañas de recolección en su predio de regeneración de Bosque de Mata Atlántico. También se agradece al Consejo Nacional de Investigaciones Científicas y Técnicas por financiar el presente trabajo.

\section{Biblografía}

AVIGLIANO, E. \& N. SCHENONE. 2015. Nymphalidae, Papilionidae and Pieridae (Insecta: Lepidoptera: Rhopalocera) from the Acaraguá river basin, Misiones, Argentina. Entomotropica 30: 81-88.

BERNICCHIA, A. \& S. P. GORJON. 2010. Corticiaceae s.1., Fungi Europaei 12. Candusso Edizioni, Alassio.

BLACKWELL, M. \& K. JONES. 1997. Taxonomic diversity and interactions of insect-associated ascomycetes. Biodiver Conser. 6: 689-699.

CALONGE, F.D. 1998. Gasteromycetes. I. Lycoperdales, Nidulariales, Phallales, Sclerodermatales, Tulostomales. Fl. Mycol. Iber. 3: 1-271.

COCKLE, K., K. MARTIN \& G. ROBLEDO. 2012. Linking fungi, trees, and hole-using birds in a Neotropical tree-cavity network: Pathways of cavity production and implications for conservation. Forest. Ecol. Manag. 264: 210 - 219.

CORNER, E. 1983. Ad Polyporaceas I. Amaruoderma and Ganoderma. Bieh. Nova Hedwigia 75: 1-182.

CORNER, E. 1984. Ad Polyporaceas II. Polyporus, Mycobonia and Echinochaete. Bieh. Nova Hedwigia 78: 1-129.

CORNER, E. 1987. Ad Polyporaceas IV. The genera Daedalea, Flavodon, Gloeophyllum, Heteroporus, Irpex, Lenzites, Microporellus, Nigrofomes, Nigroporus, Oxyporus, Paratrichaptum, Rigidoporus, Scenidium, Trichaptum, Vanderbylia and Steccherinum. Bieh. Nova Hedwigia 86: 1-265. 
CORNER, E. 1989. Ad Polyporaceas VI. The genus Trametes. Bieh. Nova Hedwigia 97: 1-197.

CUNNINGHAM, G. 1965. Polyporaceae of New Zealand. New Zealand DSIR Bulletin 164: 1-304.

DIX, N. J. \& J. WEBSTER. 1995. Fungal Ecology. Chapman \& Hall, London.

ERIKSSON, J. \& L. RYVARDEN. 1973. The Corticiaceae of North Europe. Vol. II. Fungiflora. Oslo.

FRIDMAN, J. \& M. WALHEIM. 2000. Amount, structure and dynamics of dead wood on managed forestland in Sweden. Forest Ecology and Management 131; 23-36.

GILBERTSON, R. \& L. RYVARDEN. 1986. North American Polypores. Vol. I. Fungiflora. Oslo. Norway.

GILBERTSON, R. \& L. RYVARDEN. 1987. North American Polypores. Vol. II. Fungiflora. Oslo.

HIBBETT, D. S., M. BINDER, J. F. BISCHOFF, M. BLACKWELL, P. F. CANNON, O. E. ERIKSSON, S. HUHNDORF, T. JAMES, P. M. KIRK, R. LÜCKING, H. THORSTEN LUMBSCH, F. LUTZONI, P. B. MATHENY, D. J. MCLAUGHLIN, M. J. POWELL, S. REDHEAD, C. L. SCHOCH, J. W. SPATAFORA, J. A. STALPERS, R. VILGALYS, M. C. AIME, A. APTROOT, R. BAUER, D. BEGEROW, G. L. BENNY, L. A. CASTLEBURY, P. W. CROUS, Y. C. DAI, W. GAMS, D. M. GEISER, G. W.GRIFFITH, C. GUEIDAN, D. L. HAWKSWORTH, G. HESTMARK, K. HOSAKA, R. A. HUMBER, K. D. HYDE, J. E. IRONSIDE, U. KÕLJALG, C. P. KURTZMAN, K. H. LARSSON, R. LICHTWARDT, J. LONGCORE, J. MIADLIKOWSKA, A. MILLER, J. M. MONCALVO, S. MOZLEY-STANDRIDGE, F. OBERWINKLER, E. PARMASTO, V. REEB, J.D. ROGERS, C. ROUX, L. RYVARDEN, J. P. SAMPAIO, A. SCHÜSSLER, J. SUGIYAMA, R. G. THORN, L. TIBELL, W. A. UNTEREINER, C. WALKER, Z. WANG, A. WEIR, M. WEISS, M. M. WHITE, K. WINKA, Y. J. YAO, N. ZHANG. 2007. A higher-level phylogenetic classification of the Fungi. Mycol. Res. 111: 509-47.

HLADKI, A. I. \& A. I. ROMERO. 2010. Preliminary account of Xylaria in the Tucuman province of Argentina, with a key to the known species from the northern provinces. Fungal Divers. 42: 79-96.

HLADKI, A. I. \& A. I. ROMERO. 2009. Novedades para los géneros Annulohypoxylon e Hypoxylon (Ascomycota, Xylariaceae) en la República Argentina. Darwiniana 47: 278-288.

HLADKI, A. I. \& A. I. ROMERO. 2005. Contribución al estudio de las Xylariaceae de la República Argentina. III) Nuevos registros del género Xylaria. Lilloa 42: 1-36.
JOHNSON, A. E. 2001. Las Orquídeas de Parque Nacional Iguazu. Ed. L.O.L.A, Buenos Aires.

KIERS, E. T. \& M. G. VAN DER HEIJDEN. 2006. Mutualistic stability in the arbuscular mycorrhizal symbiosis: exploring hypothesis of the evolutionary cooperation. Ecology 87: 1627-1636.

LARSEN, M. J. \& L. A. COBB-POULLE. 1990. Phellinus (Hymenochaetaceae). A survey of the world taxa. Synopsis Fungorum 3: 1-206.

LECHNER, B. E., J. E. WRIGHT \& O. F. POPOFF. 2006. New taxa and new records for Argentina of fungi from Iguazú National Park, Misiones. Fungal Divers. 21: 131-139.

LOWE, J. L. 1957. Polyporaceae of North America. The genus Fomes. Techn. Publ. Fac. Forest. State Univ. New York. 80: 1-97.

MATA HIDALGO, M. L. UMAÑA \& J. CHAVES. 2009. Documento borrador de referencia Protocolo de manejo de colecciones de hongos. Disponible en http://inbio.ac.cr/web_herbarios/web/pdf/ protocolo-hongos.pdf [Acceso: Septiembre 2015].

MORRONE, J. J. 2014. Biogeographical regionalisation of the Neotropical region. Zootaxa 3782: 1-110.

NIVEIRO, N., O. POPOFF \& E. ALBERTÓ. 2010. Contribución al conocimiento de los agaricales S.L. de la Selva Paranaense Argentina. Bol. Soc. Argent. Bot. 45: 1-2.

NIVEIRO, N., P. ZULIANI, N.A. RAMIREZ, O. POPOFF\& E. ALBERTÓ. 2014. Hongos agaricoides de las Yungas argentinas. Clave de géneros. Lilloa 51 (1): 74-86.

NORDÉN, B., M. RYBERG, F. GOTMARCK \& B. OLAUSSON. 2004. Relative importance of coarse and fine woody debris for the diversity of woodinhabiting fungi in temperate broadleaf forests. Biol.Cons. 117: 1-10

PLACCI, L. G. \& P. A. GIORGIS. 1993. Estructura y diversidad de la selva del Parque Nacional Iguazú, Argentina. Actas VII Jornadas Técnicas de Ecosistemas Forestales Nativos: uso, manejo y conservación 7: 253-267.

ROBLEDO, G. L. \& M. RAJCHENBERG. 2007. South American polypores: first annotated checklist from Argentinean Yungas. Mycotaxon 100: 5-9.

ROMANO, G. M., J. CALCAGNO, J. \& B. E. Lechner. 2013. Biodiversity of Agaricomycetes basidiomes associated to Salix (Salicaceae) and Populus (Salicaceae) plantations. Darwiniana Nueva Serie 1: $67-75$.

RYVARDEN, L. \& I. JOHANSEN. 1980. A Preliminary Polypore flora of East Africa. Fungiflora, Oslo, Norway: 1-636.

RYVARDEN, L. 1991. Genera of polypores, Nomenclature and Taxonomy. Synopsis Fungorum 5: $1-363$. 


\section{E. M. Grassi et al. - Macrohongos de la zona centro de Misiones}

RYVARDEN, L. \& R. L. GILBERTSON. 1994. European Polypores.Fungiflora, Oslo, Norway: 392-743.

RYVARDEN, L. \& A. A. R. DE MEIJER. 2002. Studies in neotropical polypores 14 . New species from the state of Paraná, Brazil. Synopsis Fungorum 15: 3469.

RYVARDEN, L. 2004. Neotropical Polypores. Fungiflora, Oslo, Norway: 1-229.

SAIBENE, C., M. CASTELINO, N. REY, J. J. HERRERA \& J. CALO. 1996. Inventario de las aves del Parque Nacional "Iguazú", Misiones, Argentina. Ed. L.O.L.A., Buenos Aires.

SILVA, V., M.V. VIGNALE, C.A. ROBLES, E. SIR. 2015. Three new records for the mycobiota Xylariaceae (Ascomycota) of Argentina. Bol. Soc. Argent. Bot. 50 (Supl.): 236-237.

SINGER, R. \& P. DIGILIO. 1951. Pródromo de la Flora Agaricina Argentina. Lilloa 25: 5-461.

SINGER, R. 1986. The Agaricales in Modern Taxonomy.4th Ed. Koeltz Scientific Books, Koenigstein.
SMITH S. E., D. J. READ. 2008. Mycorrhizal symbiosis.3rd Ed. Academic Press, London.

VAN DER HEIJDEN, M. G. A., F. M. MARTIN, M. A. SELOSSE \& I. R. SANDERS. 2015. Mycorrhizal ecology and evolution: the past, the present, and the future. New Phytologist 205: 1406-1423.

VIGNALE, M. V., E. GRASSI, G. L. ROBLEDO. 2015. New record for Rickiella edulis (Ascomycota) from Argentina. Bol. Soc. Argent. Bot. 50 (Supl.): 237237.

WRIGHT, J. E. \& A. M. WRIGHT. 2005. Checklist of the mycobiota of Iguazú National Park (Misiones, Argentina). Bol. Soc. Argent. Bot. 40: 23-44.

Recibido el 29 de diciembre de 2015, aceptado el 1 de abril de 2016 . 
